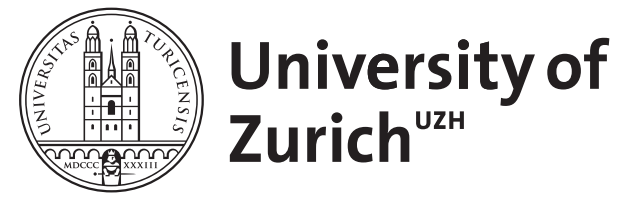

\title{
Sprachliche Oberflächen: Musterhinweise
}

\author{
Kesselheim, Wolfgang
}

DOI: https://doi.org/10.1515/9783110229301.337

Posted at the Zurich Open Repository and Archive, University of Zurich ZORA URL: https://doi.org/10.5167/uzh-56340

Book Section

Published Version

Originally published at:

Kesselheim, Wolfgang (2011). Sprachliche Oberflächen: Musterhinweise. In: Habscheid, Stephan. Textsorten, Handlungsmuster, Oberflächen. Berlin: de Gruyter, 337-366.

DOI: https://doi.org/10.1515/9783110229301.337 


\section{Sprachliche Oberflächen: Musterhinweise}

\section{Wolfgang Kesselheim (Zürich)}

1. Einführung

1.1 Problemaufriss

1.2 Hintergründe

1.3 Plan des Beitrags

2. Exemplarische Textsortenanalyse

2.1 Dezidierte Musterhinweise

2.2 Die Musterhaftigkeit der Abgrenzungs- und Gliederungshinweise

2.3 Die Musterhaftigkeit der Verknüpfungshinweise

2.4 Die Musterhaftigkeit der Themahinweise

2.5 Die Musterhaftigkeit der Funktionshinweise

2.6 Die Musterhaftigkeit der Intertextualitätshinweise

2.7 Die Musterhaftigkeit der Objekttexte

3. Ergebnisse der exemplarischen Textsortenanalyse

4. Literaturverzeichnis

\section{Einführung}

\subsection{Problemaufriss}

Die Frage, welcher Textsorte ein gegebener Text zuzuordnen ist, wird in unserem Lektürealltag nur selten zu einem Problem. Das liegt nun keineswegs daran, dass die Zuordnung von Textexemplaren zu einer Textsorte nur von akademischem Interesse wäre. Das Gegenteil ist der Fall. Da Textsorten als routinehafte Lösungen wiederkehrender kommunikativer Probleme verstanden werden können, ${ }^{1}$ bedeutet die Identifizierung einer Textsorte gleichzeitig die Identifizierung des kommunikativen Problems, das der vorliegende Text bearbeitet. Kennen wir die Textsortenzugehörigkeit, kennen wir die Zwecksetzungen, in die der Text eingebettet ist. Dann wissen wir, ob der Text in Abb. 1 dazu dient, unser Verhalten im öffentlichen Raum zu steuern, um die Sicherheit der Gebäudenutzer zu garantieren (,Keine Gegenstände in den Fluchtweg stellen!'), oder ob er uns die Möglichkeit eröffnet, bisher Unbekanntes an einem in der Nähe befindlichen Objekt zu entdecken (,Diese unbekannten Objekte sind Exemplare von Birostrina. ').

1 S. a. Luckmann (1986) und Ayaß, in diesem Band, zum Konzept der kommunikativen Gattungen. 


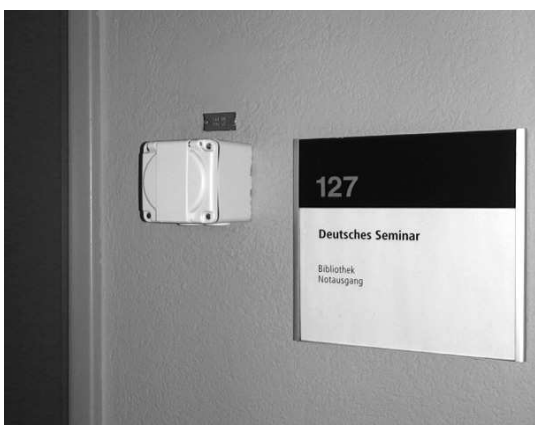

Abb. 1: Türschild Bibliothek

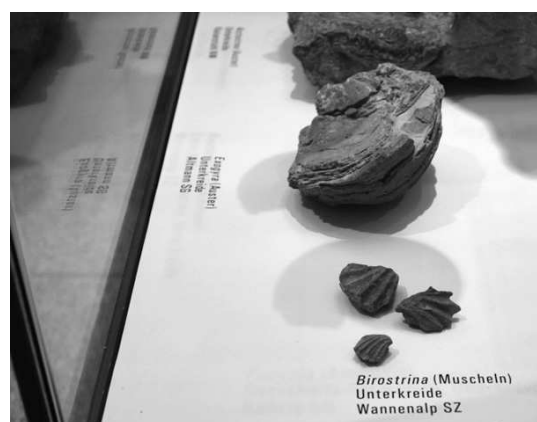

Abb. 2: Vitrine 38, Objekttext Birostrina

Ebenso können wir ausgehend von der Textsortenbestimmung Handlungsmöglichkeiten und -verpflichtungen erkennen, die sich für uns aus der Lektüre ergeben: Sollen wir das Objekt in der Nähe des Texts mit Muße betrachten und versuchen, seine Typik zu erfassen oder sollen wir uns die Lage des Objekts, auf das im Text referiert wird, für spätere Notfälle merken?

Wenn die Textsortenzuordnung also selten als Problem erfahren wird, liegt das also nicht an ihrer geringen Bedeutung für unser Alltagshandeln. Der Grund liegt vielmehr darin, dass wir eine große Expertise darin entwickelt haben, in unserem Lektürealltag Indizien auszuwerten, die uns die Zugehörigkeit eines Texts zu einer Textsorte signalisieren. Diese Indizien werden hier (nach Hausendorf \& Kesselheim 2008:23ff.) „Textsortenhinweise“ genannt werden.

Das Wort „Hinweis“ mag die Vorstellung befördern, dass Textsortenhinweise grundsätzlich metakommunikativ sind. Tatsächlich gibt es Fälle, in denen sich im fraglichen Text selbst oder in seiner unmittelbaren Umgebung ein expliziter Hinweis auf seine Klassenzugehörigkeit befindet. So kann ein Bahnfahrplan mit dem Wort „Abfahrtsplan“ überschrieben sein und ein Kassenbon kann seine Textsortenzugehörigkeit mit einer Formulierung wie „Umtausch nur mit diesem Kassenbon“ zu erkennen geben oder er kann eine andere Textsortenzugehörigkeit explizit bestreiten (,Gilt nicht als Garantiebeleg!“). ${ }^{2}$

Wie aber entsteht ein, Textsortenverdacht', wenn ein Text nicht mit solchen offenkundigen Hinweisen operiert? Hier kommen andere Hinweise ins Spiel, Hinweise, die nicht die Zugehörigkeit eines Texts zu

2 Aus Erfahrung wissen wir jedoch, dass wir uns im Alltagsleben nicht ungeprüft auf solche Selbstauskünfte verlassen dürfen. Man denke etwa an den Trick, Werbepost auf dem Umschlag mit dem Stempel „wichtige Dokumente“ zu versehen. 
einer Textsorte signalisieren ${ }^{3}$, sondern die auf andere textkonstituierende Aspekte oder „Textualitätsmerkmale“ (Hausendorf \& Kesselheim 2008) abzielen. So sind „Abgrenzungs- und Gliederungshinweise“ auf das Textualitätsmerkmal der „Begrenzbarkeit" ausgerichtet, „Verknüpfungshinweise“ auf das der „Verknüpfbarkeit", „Themahinweise“ auf die „thematische Zusammengehörigkeit“, „Funktionshinweise“ auf die „pragmatische Nützlichkeit" und „Intertextualitätshinweise“ auf das Merkmal der ,intertextuellen Beziehbarkeit“. All diese Textualitätshinweise können zu „Musterhinweisen“ werden, die für die Zuordnung eines Texts zu einer Textsorte sprechen, und zwar dann, wenn sie bei der Lektüre als musterhaft wahrgenommen werden. Die Musterhaftigkeit eines Texts kann sich aus jedem einzelnen dieser Textualitätshinweise speisen. Aus der Musterhaftigkeit seiner Abgrenzungshinweise (siehe z.B. die Musterhaftigkeit des Verkehrsschilds), aus der seiner Verknüpfungshinweise (vgl. die charakteristischen Wiederholungen einer Litanei), seiner Themahinweise (etwa auf das Thema Tod in Traueranzeigen), seiner Funktionshinweise (vgl. die vielen Elemente auf Geldscheinen, die der Bestätigung der Echtheit dienen), seiner Hinweise auf Intertextualität (der Bezug auf das rezensierte Werk in einer Rezension) oder - wie die Analysen in Abschnitt 2 illustrieren werden - ganzhaft aus einer Kombination der Musterhaftigkeit aller Textualitätsmerkmale.

Zur Entdeckung der Musterhinweise - wie generell der Textualitätshinweise - ziehen wir in unserem Lektürealltag drei unterschiedliche „Textualitätsquellen“ heran: die „Sprachlichkeit“, die „Wahrnehmung" und die „Vertrautheit“ (Hausendorf \& Kesselheim 2008:31ff.).

Zunächst erfordert die Auswertung der Musterhinweise die Berücksichtigung all dessen, was wir im Text lesen können und was wir mit Hilfe unserer Kompetenz in der deutschen Sprache aus dem Gelesenen erschließen können (Textualitätsquelle „Sprachlichkeit“).

3 „Signalisieren“ wird hier nicht terminologisch gebraucht (ebenso wenig wie die Ausdrücke „markieren“ oder „anzeigen“). Tatsächlich könnte man die unterschiedlichen Hinweise auf zwei Positionen in Bühlers bekannter Trias von „Signal“, „Symptom“ und „Symbol“ beziehen (vgl. Bühler 1934:24ff.): Metakommunikative Hinweise könnten als „Signale“ aufgefasst werden, nämlich als „Appell“ des Verfassers, den Text einer bestimmten Textsorte zuzuordnen, während die restlichen Hinweise den Status von „Symptomen“ hätten, insofern als in ihnen die Zugehörigkeit des Textexemplars zu einer Textsorte sicht- und lesbar zum „Ausdruck“ kommt. Allerdings würde eine solche Unterscheidung von „Textsortensignalen“ und „Textsortensymptomen“ möglicherweise die Tatsache verdecken, dass der Verfasser, der hier zur Textsortenzuordnung ,auffordert', eine (Re-)Konstruktion der Leser auf der Grundlage dessen ist, was sie bei der Lektüre wahrnehmen, lesen und an relevanten Lektürekontexten aktivieren können. $\mathrm{Zu}$ den Wurzeln des hier vertretenen „Hinweis“-Konzepts siehe unten, 1.3. 
Musterhinweise sind jedoch nicht auf diese Textualitätsquelle beschränkt. Sie können sich weiterhin aus dem ergeben, was alles in der Lektüresituation ,auch noch " wahrnehmbar ist (Textualitätsquelle „Wahrnehmung“). So haben wir sicherlich, noch bevor wir die Wörter „Deutsches Seminar Bibliothek Notausgang“ oder „Birostrina (Muscheln) Unterkreide Wannenalp SZ“" gelesen haben, registriert, dass die Texte in Abb. 1 und 2 aus nur wenig Wortmaterial bestehen oder aber dass es sich um gedruckte Buchstaben handelt (und nicht um von Hand Geschriebenes). Ebenso ließ sich wahrnehmen, dass sich die Texte hinter Glas befinden (durch den Leser ${ }^{4}$ nicht zu verändern!) oder dass sie auf stabile Art und Weise mit ihrer Umgebung verbunden sind (keine provisorisch angebrachte Mitteilung eines Passanten!).

Bei der Auswertung der wahrnehmungs- und sprachabhängigen Textualitätshinweise kommt im Fall der Musterhinweise notwendig eine dritte Textualitätsquelle hinzu: die „Vertrautheit". Denn die Musterhaftigkeit eines Textualitätshinweises lässt sich immer erst mit Bezug auf vorausgehende Erfahrungen mit anderen Hinweisen, anderen Texten und Handlungszusammenhängen beurteilen. So lässt sich aus der Beobachtung, dass der in Abb. 1 repräsentierte Text hinter Plexiglas angebracht ist, zwar rein wahrnehmungsbasiert ein Abgrenzungshinweis gewinnen (das Ende des Glases signalisiert das Ende des Texts!). Aber erst wenn man eine Vielzahl gleich gelagerter Abgrenzungshinweise beobachtet hat, kann man die Musterhaftigkeit dieser Abgrenzungshinweise erkennen, die auf die Benutzerlenkung in öffentlichen Gebäuden als relevanten Interpretationshintergrund verweist. Wie dieses Beispiel auch erkennen lässt, bezieht sich die Musterhaftigkeit dieser Hinweise nicht nur auf deren Form. Sie erstreckt sich gleichermaßen auf ihren Vorkommens- und Interpretationskontext, den wir mit zunehmender Vertrautheit ebenso als musterhaft erkennen können. So können Musterhinweise als contextualization cues fungieren (Gumperz 1982:130ff.), die diese Kontexte vertrautheitsabhängig als relevante Interpretationshintergründe aufrufen können.

Diese drei Quellen von Textualität gilt es bei der Textsortenanalyse im Blick zu behalten, wenn rekonstruiert werden soll, wie wir in der Alltagswelt mit Texten umgehen.

4 Bei „dem Leser“ wie auch „dem Besucher“ handelt es sich um Funktionsrollen, nicht um Personen aus Fleisch und Blut. Sie sind hinsichtlich ihrer Geschlechtszugehörigkeit nicht bestimmt. 


\subsection{Hintergründe}

Der hier vertretene textlinguistische Analyseansatz ist inspiriert von der ethnomethodologischen Konversationsanalyse. ${ }^{5}$ Zwar gibt es deutliche Unterschiede zwischen Gesprächen - dem zentralen Gegenstand der Konversationsanalyse - und Texten. So gibt es in Texten keine Wahrnehmungswahrnehmung von Textproduzenten und Rezipienten, den Rezipienten fehlt die Möglichkeit, jederzeit kommentierend und korrigierend in den Produktionsprozess einzugreifen, und schließlich ist die Überprüfung der Analysebehauptungen an den öffentlich gemachten Interpretationen der Gesprächsteilnehmer nicht möglich - ein grundlegender Validierungsmechanismus konversationsanalytischer Interpretationen (Sacks, Schegloff \& Jefferson 1978:729). Dennoch lassen sich durchaus einige der analytischen Grundprinzipien von Ethnomethodologie und Konversationsanalyse auf den Gegenstand Text übertragen. ${ }^{6}$

- Die Konversationsanalyse betont die grundlegende Gemachtheit sozialer Tatsachen. Alles, was uns in unserem Alltag als fraglos gegebene, ,natürliche' Tatsache begegnet, muss als das Ergebnis von intersubjektiven Herstellungsprozessen verstanden werden. Diesen Herstellungsprozessen versuchen Ethnomethodologie und Konversationsanalyse in der Interaktion auf die Spur zu kommen. Überträgt man diese Sichtweise auf den Untersuchungsgegenstand Text oder konkreter: Textsorten, heißt das, dass auch diese nicht einfach ,da sind' und objektiv gegebene Konturen besitzen. Vielmehr werden sie erst im Prozess der Lektüre konstruiert, und zwar indem Leser die (oben schon vorgestellten) „Textualitätshinweise“ auswerten, die sie aus dem gewinnen, was in der Lektüresituation wahrnehmund lesbar ist oder was wissensbasiert aktiviert werden kann.

- Ziel der konversationsanalytischen Untersuchungen ist es, die Alltagsmethoden, mit denen die Gesellschaftsmitglieder die sozialen Tatsachen herstellen und interpretieren, aus einer rekonstruktiven Perspektive heraus zu beschreiben (s. dazu Gülich 2001). Es gilt, „die Teilnehmerperspektive einzunehmen“, anstatt die Konstruktionsvorgänge ,von außen“ mit Begriffen zu erklären, die von einer Theorie abgeleitet sind. Auf die Analyse von Texten und Textsorten übertragen heißt das: den Alltagsmethoden auf die Spur zu kom-

5 Einführend dazu siehe Bergmann (1988) oder Hutchby \& Wooffitt (2008:11ff.).

6 Schon früh sind Texte bzw. der Umgang mit Texten von der Ethnomethodologie thematisiert worden, s. etwa Cicourel (1975) oder Garfinkel (1967), neuer: Smith (1990) oder Wolff (2006). In dem vorliegenden Beitrag geht es aber nicht darum, Texte als Gegenstand der ethnomethodologischen Konversationsanalyse zu etablieren, sondern die konversationsanalytische Analyseperspektive für die textlinguistische Analyse fruchtbar zu machen. 
men, mit denen wir die Bedeutung von Texten, ihre Funktion, Textsortenzugehörigkeit usw. konstruieren. Damit ist nun nicht die Analyse einzelner Interpretationsvorgänge realer Leser gemeint. Bei dem hier vorgestellten textlinguistischen Ansatz geht es vielmehr darum, Potenziale für bestimmte Lesarten, bestimmte Lesewege, bestimmte thematische Erwartungen usw. zu identifizieren, die von Alltagslesern bei ihrer Lektüre aktiviert werden können. ${ }^{7}$

- Die Konversationsanalyse ist strikt an dem orientiert, was in der Interaktion beobachtbar ist, was sich die Gesprächspartner gegenseitig zu erkennen geben (,,anzeigen“, s. a. Habscheid, in diesem Band). Mentale Prozesse der Interpretation, Pläne der Gesprächsbeteiligten, ihre Intentionen usw. liegen außerhalb ihres Untersuchungsbereichs. Möchte man inspiriert von der konversationsanalytischen ,Analysementalität' (Schenkein 1978:1) untersuchen, wie Texte bei der Lektüre hergestellt werden, ist es notwendig, die Analyse ganz eng auf das auszurichten, was bei der Lektüre les- und wahrnehmbar ist. In dem hier vorgestellten Modell sind dies die oben bereits angesprochenen „Textualitätshinweise“.

\subsection{Plan des Beitrags}

In dem vorliegenden Beitrag werde ich darstellen, was aus der Perspektive einer Textlinguistik, die eng an den in der Lektüresituation zugänglichen Erscheinungsformen operiert und die auf die Rekonstruktion des alltäglichen interpretativen Umgangs mit Texten abzielt, unter dem Begriff Textsorte verstanden wird und welche analytischen Konsequenzen sich aus diesem Textsortenverständnis ergeben. Um die Ausführungen nicht allzu theoretisch werden zu lassen, möchte ich das hier vertretene Modell der Textsortenanalyse ausgehend von der exemplarischen Untersuchung eines kleinen Textkorpus erläutern und illustrieren.

Bei der Textsorte, die hier analysiert werden soll, handelt es sich um die für Museumsausstellungen charakteristischen ,Schildchen', auf denen sich kurze Informationen zu den ausgestellten Objekten befinden. Eine textlinguistische Untersuchung dieser Textsorte steht meines Wissens noch aus. ${ }^{8}$ In der museologischen und museumspraktischen Literatur jedoch sind diese Texte unter Bezeichnungen wie „Objekttexte“, „Objektkennungen“ oder „Label“ immer wieder zum Thema

7 Wollte man einzelne Interpretationsvorgänge bei der Lektüre analysieren, benötigte man völlig andere Daten: Beobachtungsdaten, Daten aus experimentellen Settings, Laut-Denk-Protokolle usw. (s. a. Kesselheim (demnächst)).

8 Dawid und Schlesinger entwerfen eine Hierarchie von musealen Textsorten. Allerdings handelt es sich bei dem Buch um einen praktischen Ratgeber (Dawid \& Schlesinger (2002:35ff.)). 
geworden. Im Zentrum der wissenschaftlichen Auseinandersetzung mit den Objekttexten stand lange Zeit die Frage, ob erläuternde Texte ein unverzichtbarer Bestandteil von Museumsausstellungen sind (um dem allgemeinen Publikum einen Zugang zu dem ausgestellten Fachwissen zu ermöglichen) oder ob sie eher der eigentlichen Bestimmung des Museums im Wege stehen, nämlich einen direkten Zugang zu den authentischen Zeugnissen der Vergangenheit zu gewähren (s. etwa Tripps 1987; Graf 1985 oder Borun \& Miller 1980). Allerdings wurde diese Diskussion über weite Strecken ohne jede Bezugnahme auf konkrete Objekt-Objekttext-Kombinationen geführt und ohne den Versuch zu unternehmen, der Funktion von Objekttexten bei der Erschließung eines Objekts genauer auf die Spur zu kommen. ${ }^{9}$ Daneben gibt es eine kaum zu überblickende Ratgeberliteratur mit praktischen Handreichungen und Tipps für das Verfassen von Objekttexten (s. etwa Schneemann 2004; Dawid \& Schlesinger 2002 oder Weber 1995).

Konkret beruhen die Analysen dieses Beitrags auf einem kleinen Korpus von ca. 200 Fotografien aus dem Zürcher Paläontologischen Museum. ${ }^{10}$ Die Fotografien dokumentieren nicht nur die Texte selbst, sondern auch ihr Vorkommen in der Lektüresituation. Somit wird nicht nur der Wortlaut der Texte für die Analyse zugänglich, sondern auch (viele der) Wahrnehmungen, die dem Leser in der originalen Lektüresituation zugänglich sind und die er für seine alltagsweltliche Textsortenzuordnung heranziehen kann. ${ }^{11}$

Die Auswahl der zu analysierenden Texte beruht nicht auf einer vorgängigen Definition der Textsorte „Objekttext" (oder des Begriffs „Textsorte"). Ebenso wenig war für die Auswahl entscheidend, ob das Korpus eine Musterhaftigkeit von Texten in diesem Museum, in naturhistorischen Museen oder gar von Museumsausstellungen schlechthin repräsentiert. Die Auswahl ist lediglich durch die vorwissenschaftlich beobachtete Ähnlichkeit dieser Texte motiviert. Die Antwort auf die Frage, inwieweit sich eine einheitliche Musterhaftigkeit der Texte des Korpus feststellen lässt und in welchem Maß sich die Aussagen zur Musterhaftigkeit dieser Texte verallgemeinern lassen, kann erst der Endpunkt, nicht aber der Ausgangspunkt der Textsortenanalyse sein.

9 Eine Ausnahme bietet der mittlerweile klassische Aufsatz von Paulette McManus (1989), die untersucht, wie Besucher Objekttexte in ihre Konversation einbeziehen.

10 Sowie einem Kontrastkorpus von etwa genauso vielen Fotos aus dem Zoologischen Museum der Universität Zürich, das hier aber nur in Einzelfällen zum Einsatz kommt.

11 Trotz alledem unterscheidet sich der dreidimensionale räumliche Eindruck beim tatsächlichen Museumsbesuch von dem der Fotografie mit ihrer Zweidimensionalität, ihrem von der menschlichen Wahrnehmung abweichenden Blickwinkel, der Kadrierung usw. 
Die exemplarische Analyse der Objekttexte im Paläontologischen Museum soll demonstrieren, wie man ausgehend von der Beschreibung der Musterhaftigkeit einzelner Aspekte von Textualität Schritt für Schritt das Gesamtbild der Musterhaftigkeit einer Textsorte rekonstruieren kann. Die Musterhaftigkeit von Textsorten wird sich im Spiegel der Analysen als ein komplexes Phänomen erweisen, das nicht reduktiv durch die Aufstellung einer Texttypologie in den Griff zu bekommen ist, sondern vielmehr durch detaillierte empirische Untersuchungen, die von dem ausgehen, was bei der Lektüre konkreter Texte an Hinweisen aktiviert werden kann, und die in der Lage sind, die tatsächliche Komplexität der Textwelt in ihrer Vielfalt systematisch zu beschreiben.

Die Analyse ist entlang der weiter oben erwähnten Textualitätsmerkmale organisiert: Begrenzbarkeit, intratextuelle Verknüpfbarkeit, thematische Zusammengehörigkeit, pragmatische Nützlichkeit und intertextuelle Beziehbarkeit (s. u. 2.2-2.6). Unterabschnitt 2.7 stellt die übergeordnete Musterhaftigkeit der Textsorte „Objekttext", die sich aus der Musterhaftigkeit der einzelnen Textualitätshinweise ergibt, zusammenfassend dar. Abschnitt 3 ist schließlich den allgemeinen Folgerungen aus der exemplarischen Textsortenanalyse gewidmet: Konsequenzen für das Verständnis des Begriffs Textsorte, das Vorgehen bei der Textsortenanalyse und die Neubestimmung des Zusammenhangs von Text und Textsorte.

\section{Exemplarische Textsortenanalyse}

\subsection{Dezidierte Musterhinweise}

Bezeichnungen, mit denen Texte ihre Textsortenzugehörigkeit zum Ausdruck bringen können, sind Legion (vgl. Techtmeier 2005). Dabei steht bald die Musterhaftigkeit eines einzelnen Textualitätsmerkmals im Vordergrund (Abgrenzung: „Schild“, „Zettel“; Verknüpfung: „Erörterung", „Sonett"; Funktion: „Gebet", „Bewerbung"; usw.), bald die Musterhaftigkeit mehrerer Textualitätsmerkmale gleichzeitig (z. B. Gedenkstein: Musterhaftigkeit der Funktion Gedenken und der Abgrenzung Stein).

Bei der Häufigkeit des Vorkommens dieser Textsortenbezeichnungen im Lexikon des Deutschen ist der Befund durchaus bemerkenswert, dass sich im gesamten hier untersuchten Korpus nicht einer dieser expliziten Textsortenhinweise ${ }^{12}$ finden lässt. Textsortenbezeich-

12 Hausendorf \& Kesselheim (2008:175ff.) sprechen von „performativen Hinweisen“. 
nungen kommen hier weder als Bestandteil der Objekttexte vor (z. B. in Form einer Überschrift) noch als Referenzausdruck in anderen Texten des Museums (wie z.B. ,Detaillierte Informationen finden Sie jeweils auf den Objekttexten in unmittelbarer Nähe zu den Exponaten'). Dass dies so ist, liegt nicht etwa daran, dass eine alltagssprachliche, mit der Popularisierungsabsicht der Museumsmacher vereinbare Bezeichnung für Texte wie den untersuchten fehlte: Wer kennt nicht die sprichwörtlichen ,Schildchen' im Museum? Dass sich die Objekttexte nicht explizit zu erkennen geben, ist vielmehr ein Aspekt ihrer Musterhaftigkeit.

Allerdings gibt das Fehlen dezidierter Musterhinweise nicht schon das Signal „Das ist ein Objekttext!“, sondern verweist noch sehr unspezifisch auf die große Menge von Texten des Alltags, die uns regelhaft ohne ein klassifizierendes Etikett begegnen und deren Gemeinsamkeit nicht einfach zu bestimmen wäre: Handelt es sich darum, dass hier das Ziel der schnellen Steuerung des Lesers oder der Informationsökonomie im Vordergrund steht? Sind es Texte, die nicht das Signal geben, dass sich ihre Lektüre um der Lektüre willen lohnt? Signalisiert das Fehlen der Selbstauskunft, dass bei einer falschen Textsortenzuordnung keine nachteiligen Folgen zu erwarten sind (anders etwa als bei Textsorten wie Quittung, Mietvertrag oder Geburtsurkunde)?

$\mathrm{Da}$ also dezidierte Textsortenhinweise im hier untersuchten Korpus fehlen, muss sich die Musterhaftigkeit der Objekttexte aus anderen Textualitätshinweisen ergeben. Die Hypothese, die es in den folgenden Abschnitten im Einzelnen zu belegen gilt, ist, dass auch andere Textualitätshinweise zu Musterhinweisen werden können: Immer wenn Leser in der Realisierung einzelner Textualitätshinweise eine Musterhaftigkeit erkennen, werden diese Textualitätshinweise zu Musterhinweisen, die auf die Zugehörigkeit eines Textexemplars zu einer bestimmten Textsorten hinweisen können.

\subsection{Die Musterhaftigkeit der Abgrenzungs- und Gliederungshinweise}

Texte markieren ihre äußeren und inneren Grenzen. „Abgrenzungshinweise" zeigen an, was alles als Textbestandteil angesehen werden soll und was als Bestandteil der Umwelt, ${ }^{13}$ „Gliederungshinweise“ stellen innere Grenzen und durch diese Grenzen gebildete Untereinheiten her.

Welche Abgrenzungshinweise lassen sich in Abb. 3 erkennen?

13 Oftmals ist die Rede davon, dass ein Text seinen Anfang und sein Ende markieren müsse. In dieser Formulierung steckt aber die Vorstellung der Linearität von Texten, die nicht auf alle Arten von Texten gleichermaßen zutrifft. 


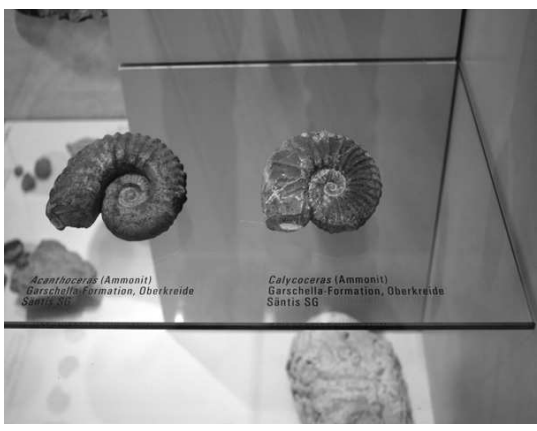

Abb. 3: Vitrine 38, Objekttexte Acanthoceras, Calycoceras

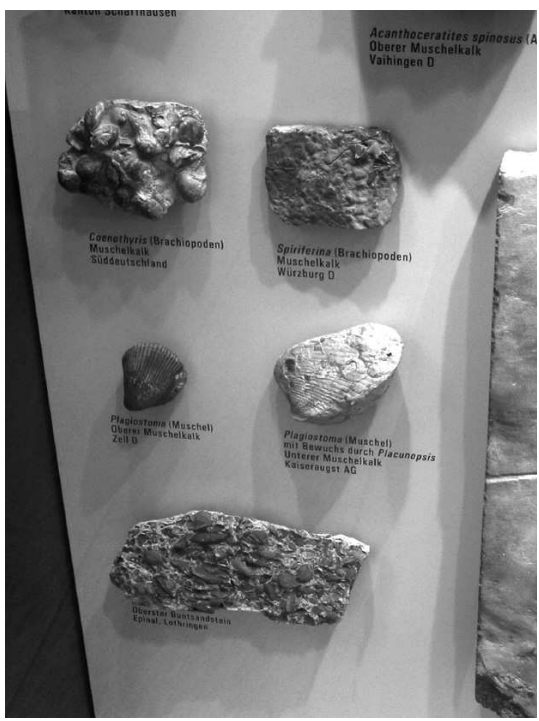

Abb. 5: Vitrine 36, unten

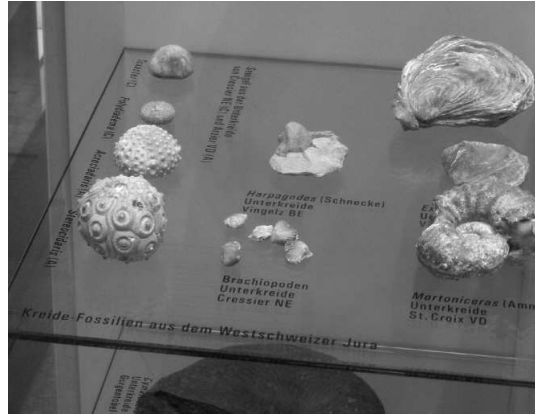

Abb. 4: Vitrine 35, Vitrinenwand, Mitte links

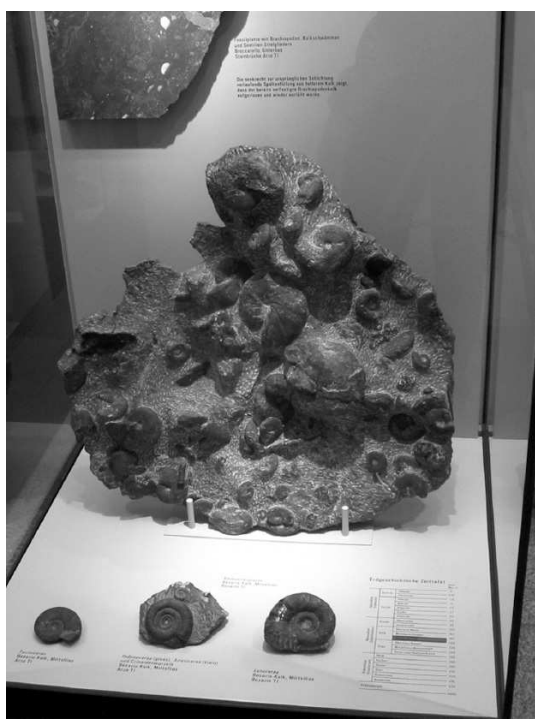

Abb. 6: Vitrine 38, oberer Glasboden, links

Sprachliche Abgrenzungshinweise fehlen hier völlig. Weder gibt es Überschriften wie ,Informationen zum Objekt', die die Erläuterungen zu den Exponaten als textuelle Ganzheiten markieren würden, noch gibt es in den Erläuterungen selbst sprachliche Ausdrücke, die auf einen Objekttext als Ganzes referierten und diesen so als Ganzheit auswiesen (etwa ,Weitere Informationen entnehmen Sie bitte den Schildern in den Vitrinen'). Auch charakteristische Anfangs- oder Endformeln, wie man sie aus anderen Textsorten kennt, lassen sich nicht finden.

Wertet man dagegen die Textualitätsquelle Wahrnehmung aus, lassen sich zahlreiche Hinweise finden, die die Außengrenzen der Objekttexte markieren. 
- Der Kontrast zwischen Zweidimensionalität der Objekttexte und Dreidimensionalität der Exponate.

- Der Kontrast zwischen den schwarzen Letraset-Buchstaben und dem durchsichtigen Untergrund, verstärkt durch die linksbündige Ausrichtung des Schriftblocks, die eine gerade Kante erzeugt.

- Die Verteilung des ,leeren Raums': Um den Schriftblock herum befindet sich weit mehr, leerer Raum' als zwischen den einzelnen Bestandteilen des Schriftblocks (Zeilen, Buchstaben). ${ }^{14}$

- Die Glasscheibe, die eine Fortsetzung des Texts jenseits des Glases zu etwas sehr Unwahrscheinlichem werden lässt.

Musterhaft sind diese wahrnehmungsbasierten Hinweise zunächst in dem Sinne, dass sie sich im gesamten hier analysierten Korpus wiederholen. Sobald man mehrere Vitrinen abgeschritten und betrachtet hat, kann man die Ähnlichkeit der einzelnen Abgrenzungshinweise erkennen und wird - das ist ein Effekt der zunehmenden Vertrautheit - auch abweichende Erscheinungsformen oder ,schlechte Fälle' auf das erkannte Muster beziehen können. Beschränkt sich die Vertrautheit nicht auf das hier untersuchte Museum, sondern speist sich aus dem Besuch zahlreicher Museumsausstellungen, erweitert das die Möglichkeiten der Entdeckung und Interpretation von Abgrenzungshinweisen. Hat man ausgehend von klaren Formen der Abgrenzungshinweise in anderen Museen (z. B. durch einen eigenen Zeichenträger wie ein Schild oder ein Glasklötzchen, s. Kesselheim 2010) eine Vertrautheit mit der Musterhaftigkeit der Abgrenzungshinweise von Objekttexten erworben, so ist man sicherlich in der Lage, auch die weniger deutlichen Abgrenzungshinweise als solche zu interpretieren, die sich in dem hier untersuchten Korpus beobachten lassen. Vor allem aber eröffnet die über den Einzelfall hinausgehende Vertrautheit die Möglichkeit, in den beobachteten Abgrenzungshinweisen einen ,Fall ${ }^{\circ}$ der museumstypischen Abgrenzung von Texten in Vitrinen zu erkennen, von der der spezifische Hinweis ausgeht, ,Das ist ein Text in einer Museumsvitrine'. Konkret: eine Abgrenzung, die durch einen , leeren Raum` signalisiert wird, der auf einer Seite durch ein in der Nähe befindliches Exponat begrenzt wird und auf einer der anderen Seiten durch eine gläserne Vitrinenwand.

Nun zu den Gliederungshinweisen. Im Fall der hier untersuchten Objekttexte werden, obwohl die Texte aus nur wenigen Wörtern bestehen, eine große Zahl von Untereinheiten signalisiert. Diese sind durch eher unauffällige Gliederungshinweise voneinander abgegrenzt. Ein grundlegender Abgrenzungshinweis ist die Unterteilung der Objekttexte

14 Oberhalb des Schriftblocks wird die leere Fläche allerdings durch ein Objekt unterbrochen: ein Verknüpfungshinweis, dem ich weiter unten nachgehen werde. 
in drei Zeilen, durch die drei Untereinheiten entstehen (Zeile 1-3). ${ }^{15}$ In jeder Zeile lassen zusätzliche Gliederungshinweise weitere Untereinheiten entstehen. So etwa in Zeile 1. Der Wechsel von kursiv zu recte und der von eingeklammert vs. nicht eingeklammert legen die Existenz zweier Positionen in Zeile 1 nahe (Abb. 3: „Acanthoceras (Ammonit)“ und "Calycoceras (Ammonit)"). Als wahrnehmungsbasierte Gliederungshinweise lassen sich auch die Kommata in Zeile 2 interpretieren, durch die zwei Untereinheiten in der Zeile erzeugt werden (z. B. „Stubensandstein, Keuper"). ${ }^{16} \mathrm{Zu}$ den durch die Wahrnehmung suggerierten Gliederungen treten einige wenige sprachliche Gliederungshinweise. So findet man beispielsweise lateinisch-griechische Ausdrücke im Wesentlichen in Zeile 1, während sie in den anderen Zeilen nicht oder nur äuBerst selten auftreten (etwa in Zeile 2 als Teil einer Epochenbezeichnung: „Oberkarbon“). Die Sprachverteilung ist dabei in den meisten Fällen deckungsgleich mit den schon durch die Wahrnehmung signalisierten Untereinheiten: s. etwa „Plagiostoma (Muschel)“. Die Sprachwahl verstärkt also den wahrnehmungsbasierten Gliederungshinweis eingeklammert vs. nicht eingeklammert. Nimmt man zur Sprachlichkeit und zur Wahrnehmung nun auch die Quelle Vertrautheit hinzu, lassen sich aus dem bei der Lektüre Wahrnehm- und Lesbaren zusätzliche Gliederungshinweise gewinnen: etwa wenn man weiß, dass in der ersten Untereinheit in Zeile 1 musterhaft ein wissenschaftlicher Name für eine Gattung oder Art enthalten ist (vgl. „Pemphix sueuri“) oder die fachliche Bezeichnung für das erläuterte Objekt (,Ammonitenplatte“) ${ }^{17}$ wenn man in der zweiten Untereinheit von Zeile 1 vertrautheitsabhängig die Einordnung des Objekts in die biologische Taxonomie erkennen kann; wenn man geologisch geschult in Zeile 2 erkennt, dass es hier nicht mehr um eine biologische Klassifikation, sondern um die Einordnung in geologische Epochen und lithostratigraphische Einheiten geht; oder schließlich, wenn man in Zeile 3 aus der Alltagsvertrautheit mit der europäischen Geographie erkennen kann, dass hier geographische Eigennamen unterschiedlicher Reichweite vorkommen.

Weitet man das Korpus aus, so erweist sich die Untergliederung der Objekttexte in drei Zeilen mit einer festen Anzahl von Untereinheiten als ein lokal begrenztes Muster. In der Form der Gliederungshinweise (also in der Untergliederung durch Zeilenumbruch, Kursivschrift und

15 Eine Abweichung von der 3-Zeilen-Regularität gibt es nur, wo die erste Zeile aufgrund Überlänge umgebrochen wird, vgl. Abb. 5: „Plagiostoma (Muschel)/mit Bewuchs durch Placunopsis/Unterer Muschelkalk/Kaiseraugst AG“.

16 Kommata werden hier der Quelle „Wahrnehmung“ zugeschlagen. Die dahinter stehende Überlegung ist, dass auch ein Leser, der des Deutschen nicht mächtig ist, die Gliederungswirkung der Kommata in Zeile 2 erfassen kann.

17 Natürlich erlaubt dieses Wissen auch eine Auswertung als Themahinweis; s. u. 2.4. 
den Einsatz von Klammern zur Signalisierung der Übergänge zwischen Fach- und Alltagssprache) liegt jedoch eine Musterhaftigkeit, die auf Objekttexte allgemein verweist.

Die Objekttexte unseres Korpus konstituieren sich also durch eine ganze Reihe von Abgrenzungs- und Gliederungshinweisen als unabhängige textuelle Einheiten mit einer differenzierten Binnenstruktur. Aus dem Material lassen sich jedoch ebenso Hinweise gewinnen, die die bisher analysierten Grenzziehungen infrage stellen und für die Berücksichtigung größerer Lektüreeinheiten sprechen. Das beginnt bereits damit, dass viele Texte in dem hier untersuchten Korpus auf einen durchsichtigen Regalboden aufgebracht sind (s. etwa Abb. 3 oder 6). Während man einen dieser Texte liest, nimmt man notwendigerweise auch die Existenz anderer, gleich gestalteter Texte wahr, die sich auf anderen Ebenen der Vitrine befinden. Der Einzeltext - so das Signal, das von der gleichzeitigen Präsenz im Gesichtsfeld des Betrachters ausgeht - ist in eine Menge ähnlicher Texte eingebunden. Die Durchsichtigkeit des Regalbodens weist folglich auf eine Ganzheit hin, die über die Ganzheit der einzelnen Objekttexte hinausgeht: eine „Textsammlung" (Hausendorf \& Kesselheim 2008:44ff.) ähnlich den einzelnen Gedichten einer Anthologie, den einzelnen Einträgen eines Gästebuchs oder den einzelnen Karteikärtchen einer Vokabelsammlung.

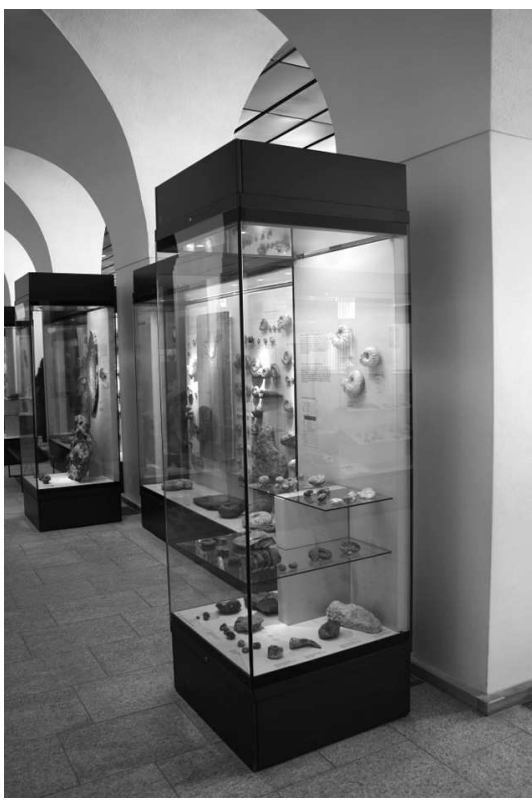

Abb. 7: Vitrine 38, Gesamtansicht
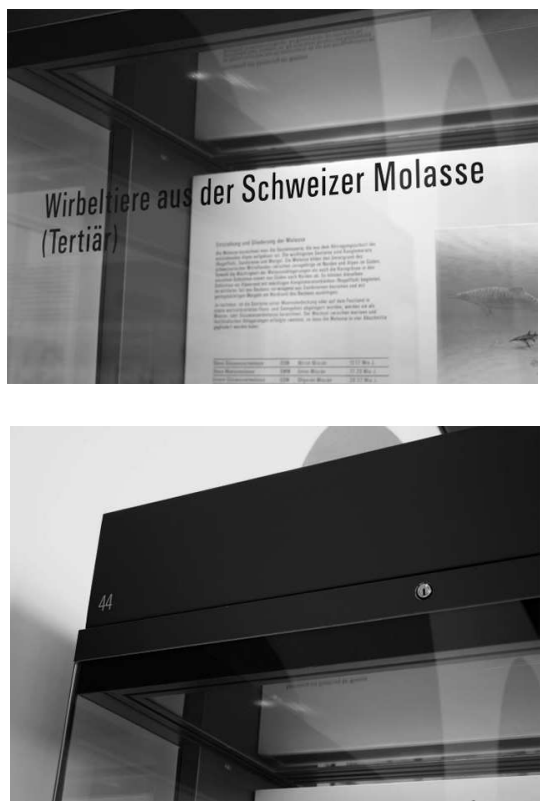

Abb. 8a, 8b: Vitrine 44, Vitrinenüberschrift und Vitrinennummer 
Die äußeren Grenzen dieser Textsammlung werden durch spezielle Ganzheitshinweise signalisiert: den abgeschlossenen Glaskörper, die Beleuchtung, die sich gleichmäßig über alle Texte und Exponate im Innern der Vitrine erstreckt und sie gemeinsam von ihrer dunkleren Museumsumgebung abhebt, die Vitrinenüberschrift (Abb. 8a) und schließlich die Nummer auf dem die Vitrine nach oben abschließenden Metallrahmen, die eine Referenz auf die Vitrine als Ganzes ermöglicht (Abb. 8b). Nach innen hin präsentiert sich die Textsammlung Vitrine als komplexe Anordnung von wahrnehmungsbasierten Gliederungshinweisen: vgl. die Anordnung der Objekte auf verschiedenen Regalböden (Abb. 3, 5 oder 7), die unterschiedliche Ausrichtung der Schrift (Abb. 5) usw.

Der einzelne Objekttext, so ist aus den mehrfach gestuften Ganzheits- und Gliederungshinweisen zu folgern, präsentiert sich zwar einerseits als abgeschlossene textuelle Einheit (mit einer privilegierten Beziehung zu dem in der Nähe befindlichen Objekt, s. u. 2.3), andererseits aber als Teil einer den Einzeltext umfassenden Textsammlung, deren äußere Grenzen durch die Glaswände der Vitrine markiert werden. Weit davon entfernt, ein , unschuldiges', sprich: für die Kommunikation irrelevantes Möbelstück zu sein, präsentiert sich die Vitrine hier als ein Ensemble von Ganzheits- und Gliederungshinweisen, das bei der Analyse unseres Umgangs mit Objekttexten mit berücksichtigt werden muss.

\subsection{Die Musterhaftigkeit der Verknüpfungshinweise}

Verknüpfungshinweise sind dafür verantwortlich, dass wir ein lesbares Etwas als sinnvolle Abfolge einzelner Elemente, also als etwas ,Gewebtes' wahrnehmen (wie die bekannte Etymologie des Wortes Text lautet). Als Verknüpfungshinweis kann alles dienen, was in der Lage ist, einen Zusammenhang zwischen verschiedenen Bestandteilen eines Texts nahezulegen. Dazu gehören Wiederholungen, die - unabhängig von ihrer Bedeutung - die Existenz einer Verbindung zwischen den identischen Elementen suggerieren, anaphorische oder kataphorische Textbezüge, die auf schon Gelesenes oder noch zu Lesendes als relevante Interpretationshintergründe verweisen, Formen der Satzkonnexion und schließlich eine große Bandbreite von sprachlichen Formen, die auf das Vorliegen globaler Verknüpfungsstrukturen hinweisen wie etwa Narration, Argumentation usw. (s. die Beiträge von Kotthoff und Reisigl, in diesem Band).

Fahndet man im Objekttextekorpus nach den sprachlichen Verknüpfungshinweisen, die bisher im Mittelpunkt der textlinguistischen Forschung standen, wird man kaum fündig. „Transphrastische“ Verknüpfungshinweise (wie Anaphern oder Satzkonnektoren) fehlen voll- 
ständig, ${ }^{18}$ und selbst die Wiederholung von Wörtern oder Wortbestandteilen, die am wenigsten anspruchsvolle Form von Verknüpfungshinweisen, kommt nur äußerst selten vor. ${ }^{19}$

Häufiger sind wahrnehmungsbasierte Verknüpfungshinweise. Auf Abb. 5 sind einige dieser Hinweise zu erkennen, die zum Eindruck eines zusammenhängenden ,Schriftblocks' beitragen. So ist beispielsweise der Zeilenabstand zwischen den Zeilen des Objekttexts deutlich geringer als der Abstand zu den Zeilen anderer, oberhalb oder unterhalb des analysierten Texts befindlicher Texte, und die linksbündige Anordnung der Zeilen unterstreicht die Tatsache ihres Zusammengehörens zusätzlich. Besonders auffällig markiert wird jedoch, wie auf Abb. 5 gut zu sehen, die Verknüpfung des Objekttexts zu je einem benachbarten Objekt. ${ }^{20}$ Musterhaft geschieht das durch die geringe Distanz zwischen Schriftblock und Objekt (wobei ,gering“ bedeutet, dass das Objekt näher an ,seinem" Text ist als an jedem anderen Text in der Vitrine). Häufig wird dabei das Objekt so positioniert, dass sein linker Rand die Kante fortsetzt, die durch die Linksbündigkeit des Schriftblocks erzeugt wird. ${ }^{21}$ Ein wichtiger Verknüpfungshinweis geht auch von der Ausrichtung des Texts aus. Auf den Text beziehbar ist nur ein Objekt, das man zeitgleich mit der Lektüre des Textes lesen kann. Sichtbar wird die Verknüpfungswirkung der Textausrichtung etwa in Abb. 5, wo die Texte der linken Seite offensichtlich auf die Seeigel zu beziehen sind, die sich durch das linke Vitrinenglas am besten betrachten lassen.

Wieder wirkt die Vertrautheit mit den Hinweisen derart, dass wir die Verknüpfung auch in ,schlechten Fällen' erschließen können, etwa im Fall der Brachiopoden in Abb. 5. Hier ist offensichtlich einer der Brachiopoden aus der halbkreisförmigen Anordnung , ausgebrochen und annulliert gleich zwei Verknüpfungshinweise auf seinen relevanten Objekttext: Zum einen ist der Abstand zumindest eines Brachiopoden zum relevanten Informationstext größer als der zum weiter oben in der Vitrine befindlichen Text, und zum anderen verletzt er die musterhafte

18 Die Konjunktion und kommt zwar vor, allerdings nicht als Satzkonnektor (z. B. ,Andrias scheuchzeri, Riesensalamander/Platte und Gegenplatte").

19 Beispiele sind die Wiederholung von -kalk in „Trochitenkalk, Muschelkalk“ oder des Ortsnamens Besazio in „Besaziokalk ... Besazio“.

20 Manchmal kommentiert ein Objekttext auch eine Mehrzahl von Objekten. Diese sind dann so angeordnet, dass der Abstand unter ihnen deutlich kleiner ist als der Abstand zu anderen Objekten in der gleichen Vitrine.

21 Diese Musterhaftigkeit ist in Vitrinen mit vielen kleinteiligen Objekten deutlicher ausgeprägt als in Vitrinen mit wenigen, großen Objekten. In Letzteren ist es offensichtlich nicht notwendig, die Verknüpfung zusätzlich hervorzuheben. Die unmittelbare Nachbarschaft von Schriftblock und Exponat reicht hier offensichtlich aus, um die Verknüpfung augenfällig werden zu lassen. 
Anordnung der Objekte in der Verlängerung der linken Kante des Schriftblocks.

Dehnt man das Korpus auf eine größere Zahl von Museen aus, wird auch offenbar, dass es sich bei der Verknüpfung von Schriftblock und Objekt um einen zentralen Aspekt der Musterhaftigkeit von Objekttexten handelt. Das legt schon die große Zahl von verschiedenen Verknüpfungshinweisen nahe, die sich im Laufe der Entwicklung der musealen Kommunikation herausgebildet hat (s. z. B. Kesselheim 2009):

- übereinstimmende Nummern oder Buchstaben auf Text und Exponat;

- Fotos oder Umrisszeichnungen der Exponate auf dem Textträger neben dem Schriftblock;

- Pfeile, die Text und Exponat(-teile) verbinden;

- die gemeinsame Beleuchtung durch eine Spotlampe;

- usw.

Diese Musterhaftigkeit der Textverknüpfung weist erneut über den Objekttext als oberste Analyseeinheit hinaus. Sie spricht für eine Textanalyse, bei der der Objekttext als Teil eines größeren kommunikativen Arrangements aufzufassen ist: ein Arrangement, das aus dem Objekttext und einem je spezifischen, in der Nähe befindlichen Objekt besteht.

Auch die oben analysierten sprachlichen Verknüpfungshinweise sprechen für diese Ausweitung der Textsortenanalyse. Fasst man nämlich nicht mehr den einzelnen Objekttext als Obergrenze der Textanalyse auf, lassen sich auf einmal eine große Zahl von sprachlichen Verknüpfungshinweisen (v. a. Wiederholungen) identifizieren. Während der einzelne Objekttext nur mangelhaft verknüpft zu sein scheint, erweisen sich die Objekttexte als hochgradig verknüpft, wenn man sie im Zusammenhang mit den anderen Texten und den Objekten (!) in der Vitrine betrachtet. So sind beispielsweise schon in dem kleinen Vitrinenausschnitt, der auf Abb. 5 zu erkennen ist, die Wörter „Muschel“ und „Muschelkalk“ sowie die verschiedenen als Muscheln erkennbaren Exponate über den Verknüpfungshinweis Wiederholung verbunden. ${ }^{22}$ Beobachtungen wie diese sprechen dafür, in der „Textsammlung“ Vitrine die Einheit zu sehen, auf die sich die Textsortenanalyse von Objekttexten beziehen muss.

22 Semiotisch ist es gewiss problematisch davon zu sprechen, ein Objekt „wiederhole“ ein Wort oder ein Wort ein Objekt. Hier geht es aber lediglich darum, ein einfaches Etikett für die Verknüpfungswirkung zu finden, die aus der gemeinsamen Präsenz von Objekt und verbaler Referenzform entsteht. Diese Wirkung lässt sich im Übrigen empirisch nachweisen, wenn man reale Rezeptionsvorgänge bei der Betrachtung von Vitrinen untersucht (Kesselheim (demnächst)). Offenbar wird das Vorkommen eines Wortes in einem Objekttext als Aufforderung interpretiert, das Referenzobjekt in der Vitrine zu suchen. 


\subsection{Die Musterhaftigkeit der Themahinweise}

Mit „Thema“ ist hier ein Zusammenhang gemeint, der durch die wiederholte Referenz auf einen als identisch oder als überlappend verstandenen Aspekt der Welt entsteht, seien es nun Personen, Objekte, Sachverhalte, zeitliche oder räumliche Koordinaten. Dabei geht es nicht darum, von irgendeinem äußeren Standpunkt aus zu bestimmen, ob die Textreferenzen, wirklich' auf ein und denselben Weltausschnitt gerichtet sind. Es geht um die Art und Weise, wie die Referenzen im Text selbst präsentiert werden: als Referenz auf einen gleich bleibenden Weltausschnitt, als Referenz, die auf vorausgehenden Referenzformen aufbaut, oder als Referenz, die auf einen gänzlich neuen Weltausschnitt gerichtet ist. Klare Themahinweise ergeben sich beispielsweise aus dem aufeinander folgenden Auftreten von Wörtern, die über lexikalisierte semantische Beziehungen miteinander verbunden sind (Synonymie, Hypo- oder Meronymie etc.) oder über mit Wörtern verbundenen konventionellen Frames und Scripts. Themahinweise ergeben sich aber auch aus den wesentlich schwieriger zu formalisierenden, von den anderen Lexemen des Texts abhängigen Isotopieebenen. Wenn Themahinweise auch oftmals weniger deutlich an der ,Textoberfläche' zu erkennen sind als etwa Verknüpfungshinweise, ist die thematische $\mathrm{Zu}$ sammengehörigkeit doch kein Phänomen, das nur im Geist des Lesers oder des Autors anzusiedeln wäre. Auch das Textthema muss ausgehend von dem rekonstruiert werden, was in der Lektüresituation sinnlich wahrnehmbar oder aus der Vertrautheit mit den Lektürekontexten aktivierbar ist.

In unserem Korpus von Objekttexten bleiben die reichen Möglichkeiten der Sprache, mit grammatischen Mitteln auf die Einführung, Fortführung oder Beendigung thematischer Stränge hinzuweisen (etwa durch die viel untersuchte Pronominalisierung oder durch das Zusammenspiel von kataphorischem und anaphorischem Artikel), ungenutzt. ${ }^{23}$ Auch explizite oder gar metakommunikative Hinweise zur Einführung (Überschriften, die Verwendung von Wörtern wie Thema, Gegenstand, Frage usw.) oder Beendigung eines Themas fehlen (,Soviel zu den Ammoniten'). Die Hauptlast bei der Konstitution des Textthemas tragen lexikalische und semantische Hinweise auf Themabeibehaltung und -entwicklung.

Die Themabeibehaltung wird beinahe ausschließlich über zwei Typen von Hinweisen signalisiert: Rekurrenz und Ellipsen. Rekurrenz,

23 Das Auftreten des anaphorischen Artikels beispielsweise ist auf Objektgruppentexte oder übergeordnete Vitrinentexte beschränkt, und auch dort lassen sie sich kaum als Themahinweis interpretieren (vgl. etwa „Seeigel aus der Unterkreide von Cressier NE (C) und Arzier VD (A)“ oder „Kreidefossilien aus dem Westschweizer Jura“). 
also die Wiederholung gleicher Wortformen und -gruppen, begründet eine gewisse Erwartung, dass das zuvor etablierte Thema unverändert fortgeführt wird, dass der Text also weiterhin auf den zuvor konstituierten Ausschnitt von Welt verweist. Vgl. etwa: „Trochytenkalk, Muschelkalk“ oder „Platte und Gegenplatte“. Doch ist die Rekurrenz nur ein relativ schwacher Themabeibehaltungshinweis. Denn die Wiederholung eines Wortbestandteils ist kein Garant dafür, dass das Thema , wirklich “ konstant bleibt. So könnte „Platte und Gegenplatte“ zum Ausgangspunkt für zwei kontrastierende Unterthemen werden (Daneš 1970). ${ }^{24}$

Während die Rekurrenz relativ spärlich vertreten ist, kommen Ellipsen in den untersuchten Texten häufig vor. Allerdings handelt es sich nur um einen ganz konkreten Typ von Ellipsen, und zwar um Ellipsen im Rahmen von Teil-Ganzes-Beziehungen (s. u.): etwa in „Diplocynodon (Krokodil), Schädel und Rückenpanzerplatten“. Körperteile sind ja immer Körperteile von einem Lebewesen. Daher versteht man hiermit, dass es sich um den Schädel und Panzerplatten von Diplocynodon handelt. In der Tatsache, dass diese Information hier offensichtlich weggelassen werden kann, liegt der Themabeibehaltungshinweis: Es geht weiterhin um das Krokodil Diplocynodon, auch wenn (oder gerade weil) diese Besitzrelation hier nicht ausgedrückt wird.

Substitutionen - die Ersetzung eines Referenzausdrucks durch einen koreferenten anderen - stehen auf der Grenze zwischen Themabeibehaltungs- und Themaentwicklungshinweisen. Denn durch jede neue Referenzform wird ein Referenzobjekt auf eine neue Art und Weise in den Blick genommen. Die neuen Perspektiven, die durch den neuen Referenzausdruck entstehen, bieten immer auch einen Ansatzpunkt für eine thematische Verschiebung. Eines der zahlreichen Beispiele aus dem Korpus: „Bonebed (Knochenschicht)“. Häufiger noch als die Substitutionen sind in den Objekttexten Hinweise auf Themaentwicklung, die sich aus der lexikalischen Information der beteiligten Wörter ergeben. Konkret: Wortpaare, die sich in einer lexikalischen Relation von Hyponymie oder Meronymie befinden. Beliebige Beispiele für eine Themaentwicklung durch Hypo- und Hyperonymierelationen sind „Coeno-

24 Dass sich die Leistung der einzelnen kommunikativen Erscheinungsform für das Textthema nicht kontextfrei bestimmen lässt, mag als Schwäche der Analyse erscheinen. Tatsächlich befindet sich der Leser aber in der gleichen Lage: Zu jedem Zeitpunkt der Lektüre muss er aufgrund des schon Gelesenen und des im Text Signalisierten Hypothesen darüber entwickeln, in welche Richtung sich das Thema entwickeln wird und was sich als das zentrale Textthema herausstellen wird. Dabei ist es sicherlich nichts Ungewöhnliches, wenn er seine thematischen Erwartungen mit Fortschreiten der Lektüre an die jeweils neue Lage anpassen muss. Deshalb muss „Thema“ dynamisch gedacht und analysiert werden (vgl. etwa den Quaestio-Ansatz von Klein \& von Stutterheim 1981). 
thyris (Brachiopoden)“ oder „Trigonodusdolomit, Muschelkalk“, Beispiele für Teil-Ganzes- und Ganzes-Teil-Relationen z. B. „Schuppen ... Fischen" oder "Schachtelhalm ... Stammes“.

Diese Besonderheit der Themaentwicklung stellt eine weitere prominente Facette der Musterhaftigkeit von Objekttexten in naturhistorischen Museen dar. Denn die Musterhaftigkeit der Themaentwicklung entlang von Hypo- und Hyperonymiebeziehungen spiegelt die penible Klassifikationsarbeit wider, die Bestrebung, jedes einzelne Objekt in eine hierarchisch organisierte Welt von Objekten einzusortieren, die für die Paläontologie als Wissenschaft zentral ist. Nirgends tritt die Definitionsarbeit, die in den Objekttexten geleistet wird, deutlicher zu Tage als in dieser Organisation der Themaentwicklung entlang gestufter Klassifikationssysteme. Gleichermaßen grundlegend für die Musterhaftigkeit der Objekttexte ist die Themaentwicklung durch Teil-Ganzes(„Schuppen ... Fischen") oder Ganzes-Teil-Relationen (,Schachtelhalm ... Stammes"). In ihr findet die Thematik des Fundstücks ihren Ausdruck, dessen Zuordnung zu (postulierten) Ganzheiten die zweite Kernaktivität der Paläontologie darstellt.

Wenn man - wie schon durch die Abgrenzungs- bzw. Gliederungshinweise sowie die Verknüpfungshinweise suggeriert - nicht den einzelnen Objekttext, sondern die Gesamtheit der Texte etwa einer Vitrine bei der Analyse berücksichtigt, explodiert buchstäblich die Zahl der Themahinweise durch Rekurrenz, Substitution usw. Liest man eine größere Zahl von Texten einer Vitrine, kann man mit der dadurch entstehenden lokalen Vertrautheit auch nicht lexikalisch kodierte Relationen wie etwa Frames oder Isoglossieebenen als Themahinweise entdecken. Basierend auf der Vertrautheit erschließen sich in den hier untersuchten Objekttexten die drei Frames Lebewesen, Alter, Ort als die zentralen Bedeutungsdimensionen, um die alle Texte kreisen und die in ihrer Kombination einen ziemlich genauen Eindruck davon geben, was ein paläontologischer Fund ist: ein Lebewesen (als Ganzes, Teil oder Spur), das einem bestimmten Erdalter zuzuordnen ist und das im Moment seines Gefundenwerdens mit einem bestimmten Ort verbunden ist, in dessen alternativen Benennungsmöglichkeiten mit Hilfe moderner (Ort, Länder- oder Kantonsbezeichnung u. ä.m.) oder erdgeschichtlicher/lithostratigraphischer Bezeichnungen sich der spezifische Doppelcharakter des Fundstücks als etwas Gewesenem und heute Betrachtbarem spiegelt. ${ }^{25}$ Eine weitere Besonderheit der hier

25 Die Bedeutung des Ort- und Alter-Frames kann man sehr schön im Kontrast zu den Objekttexten im benachbarten Zoologischen Museum erkennen (das in einem parallelen Korpus dokumentiert worden ist), in denen diese Frames - bei ganz ähnlichen Exponaten - gerade nicht vorkommen. 
beschriebenen Kombination semantischer Frames ergibt sich erst, wenn man über die lokale Vertrautheit mit der betrachteten Vitrine hinausgeht und sich geologisches und paläontologisches Wissen aneignet. Dann ist eine weitere Verbindung des Orts-Frames mit dem Zeitframe zu konstatieren, insofern als die Bezeichnung der Fundschicht (Litostratigraphie) mit der des Fundalters (Chronostratigraphie) auf spezifische Art und Weise miteinander verwoben ist.

Auch die Wahrnehmung als Textualitätsquelle lässt eine Ausdehnung der Analyse über die Grenzen der Schriftlichkeit hinaus als notwendig erscheinen. Denn wenn man nur das Geschriebene als Text analysiert, bleiben gerade diejenigen thematischen Hinweise, die dem Besucher als allererstes ins Auge springen, von der Analyse ausgeschlossen: Die bizarren Formen der Museumsobjekte, in denen wir vertrautheitsbedingt Fossilienplatten, Skelette und Ähnliches mehr erkennen können und die uns selbst dann einen klaren Hinweis auf die behandelte Thematik geben, wenn wir nicht einen der hier untersuchten Objekttexte lesen. Vertrautheitsbasiert kann man, wenn man das Korpus über die hier untersuchten Texte ausdehnt, sowohl aus den wiedererkennbaren Formen als auch aus ihrer wiedererkennbaren Anordnung - viele dreidimensionale Objekte auf engem Raum hinter Glas übereinander gestapelt, durchdrungen von Schriftblöcken unterschiedlicher Größe und Gestaltung - eine thematische Erwartung ableiten: „Das ist ein naturhistorisches Museum“.

\subsection{Die Musterhaftigkeit der Funktionshinweise}

Funktionshinweise sorgen dafür, dass wir einen Text als Zusammenhang von kommunikativen Handlungen mit einer spezifischen pragmatischen Nützlichkeit wahrnehmen. Textsorten stehen in besonders enger Verbindung mit der pragmatischen Nützlichkeit eines Texts: Zur Durchführung immer wiederkehrender kommunikativer Aufgaben haben sich spezifische Textsorten ausgeprägt, deren „Texthandlung“ genau in der Bearbeitung dieser Aufgaben liegt. Texthandlungen sind deshalb

eine Art Kristallisationspunkt für Textsorten: In ihnen nehmen Ausprägungen einer spezifischen Form von Textnützlichkeit musterhaft Gestalt an. Texthandlungshinweise und Textsortenhinweise gehen also grundsätzlich Hand in Hand (Hausendorf \& Kesselheim 2008:162).

Erkennt man die Texthandlung, kann man von ihr auf die vorliegende Textsorte schließen und umgekehrt. Zum Identifizieren der Texthandlung ist man auf eine eigene Klasse von Musterhinweisen angewiesen, die „Hinweise auf Texthandlungen“ (ebd.:161ff.). Diese Hinweise können expliziter Natur sein („Kommentar“, „Widerruf“, „hiermit erkläre ich eidesstattlich ..."). Sie können sich aber auch aus der Musterhaftigkeit der Art und Weise ergeben, mit der sich die Texthandlung aus all- 
gemeineren Textfunktionen, den „textuellen Grundfunktionen“ zusammensetzt (ebd.:143ff.): dem Bezug auf Welt („Darstellung“), dem Bezug auf den Autor („Beleg“) oder den Leser (,Steuerung“), die Beziehung zwischen Autor und Leser (,Kontakt"), der Beziehung zwischen dem Text und seinem Code („Reflexion“) und schließlich der selbstreflexive Bezug des Texts zu seiner Form („Unterhaltung“). ${ }^{26}$ Erkennt man in den Hinweisen auf diese textuellen Grundfunktionen eine wiedererkennbare ,Gestalt', kann man sie also auf eine Musterhaftigkeit beziehen, dann gestattet das den Schluss von diesen Hinweisen auf die Textsortenzugehörigkeit eines Textexemplars.

Die Musterhaftigkeit der Textfunktionen ist allerdings nicht auf die Ebene der textuellen Grundfunktionen und die der Texthandlungen beschränkt. Ein wichtiger Aspekt der pragmatischen Nützlichkeit von Texten ist ihre Einbettung in großräumige gesellschaftliche Funktionsbereiche wie Wissenschaft, Kunst oder Religion mit ihren dazugehörigen Leitunterscheidungen und Zwecksetzungen, auf die eigene Hinweise abzielen (s. u.).

Die Beispielanalyse der Textfunktionen nimmt ihren Ausgang bei den textuellen Grundfunktionen.

In den Objekttexten hat die Darstellungsnützlichkeit absoluten Vorrang. Die Texte bestehen fast ausschließlich aus Nomina, deren Leistung der referenziellen Bezugnahme auf Welt für die Objekttexte prägend ist. $^{27}$ Deutlich weniger augenfällig sind Hinweise auf die Grundfunktion der Reflexion, also auf die Thematisierung der eigenen Ausdrucksmittel. Reflexionshinweise sind die im Abschnitt zu den Abgrenzungshinweisen bereits erwähnten Klammern und die Unterscheidung von kursiv und recte, die den Gegensatz von Fachsprache und Alltagssprache sichtbar macht. Beispiele wie „Birostrina (Muscheln)“ (Abb. 2), in dem der eingeklammerte Ausdruck die alltagssprachliche Übersetzung des vorausgehenden fachlichen Terminus ist, illustrieren die typische Verbindung, die die Darstellungs- und die Reflexionsnützlichkeit in den Objekttexten eingehen: Die Reflexion ist hier an das Ziel der Vermittlung eines Fachvokabulars geknüpft. ${ }^{28}$ Wei-

26 In diesen textuellen Grundfunktionen kann man unschwer den Reflex der Jakobson'schen Sprachfunktionen erkennen (vgl. Jakobson 1972).

27 Wenn es auch Referenz auf Zeitpunkte und -abschnitte gibt, so führt das dennoch nicht dazu, dass sich eine im weitesten Sinne narrative Verknüpfung ausbildet. Denn die Referenz auf Zeitbegriffe dient hier nicht der zeitlichen Verankerung der Aussagen (also nicht: ,Das war in der Kreidezeit ein Nautilide', sondern: ,Das ist (= ein Präsens der allgemeinen Gültigkeit) ein Nautilide aus der Kreidezeit').

28 Die für wissenschaftspopularisierende Texte in der Presse charakteristische Betonung der textuellen Grundfunktion des Unterhaltens ist in den Objekttexten dagegen auffallend abwesend. Hinweise auf die Unterhaltungsfunktion ergeben sich lediglich aus den Objekten, ihrer kunstvollen Anordnung und aufwändigen Präsentation. 
tere Hinweise auf die Texthandlung ergeben sich aus der Wahrnehmung. Objekttext und Objekt sind so angeordnet, dass sie gemeinsam wahrgenommen und gelesen werden können. Daraus ergibt sich das Signal, dass der Objekttext zusammen mit dem Exponat rezipiert werden soll. ${ }^{29}$ Auf das Lesen als eine Tätigkeit, die im Vorbeigehen vollzogen werden soll, weisen die Platzierung von Objekten und Objekttexten relativ zu den Glaswänden der Vitrinen hin, aber auch die Größe der Schrift und die auffallende Kürze der sprachlichen Ausdrucksformen. Nicht nur fehlen Verben fast durchgehend, auch sind Abkürzungen (für Länder, Kantone, Fundstätten oder erdgeschichtliche Epochen) ausgesprochen häufig und die wichtige Verbindung des Texts zu ,seinem ${ }^{6}$ Exponat wird nur in Ausnahmefällen sprachlich ausgedrückt. ${ }^{30}$

Die Beobachtungen zur Musterhaftigkeit der Hinweise auf textuelle Grundfunktionen zusammengenommen, lässt sich die spezifische Texthandlung der Objekttexte wie folgt beschreiben: das kommunikative Problem, auf das die Textsorte der Objekttexte reagiert, ist die Aufgabe, Objekte wissenschaftlich zu klassifizieren und zu benennen (wobei der Leser in das paläontologische Fachvokabular geführt wird), und diese Informationen einem Leser zugänglich zu machen, der im Moment der Lektüre das relevante Objekt von außerhalb der Glasvitrine betrachtet.

Die Einbettung der Objekttexte in den gesellschaftlichen Funktionsbereich der Wissenschaft mit der ihr eigenen Terminologisierung und ihrem charakteristischen Bestreben nach Effizienz und Präzision des Ausdrucks leistet schließlich die schon erwähnte sprachliche Kürze in Zusammenhang mit der durchgehenden Verwendung von Fachsprache. Allerdings beginnt die Einbettung der Objekte in die Welt der Wissenschaftstexte nicht erst bei der Lektüre des ersten Objekttexts. Hinweise auf die Wissenschaft als relevanten Interpretationshintergrund erhält der Besucher schon, wenn er das Museum noch gar nicht betreten hat (s. Abb. 9-10): Das Universitätslogo auf dem Banner über der Eingangstüre und auf den flankierenden Stelen, die mehrfache Wiederkehr des Worts „Museum“, das auf eine Institution des Wissenschaftssystems verweist und das durch seine prominente Platzierung im Eingangsbereich eine Gültigkeit für das gesamte Gebäude beansprucht.

29 Und zwar durch einen Leser, der den Text durch das Glas der Vitrine betrachtet. Dass die Anbringung hinter Glas zur Beschreibung des Musters der Texthandlung dazugehört, wird deutlich, wenn man den Kontrastfall einer Fossilienplatte im gleichen Museum betrachtet, die nicht von Glas umgeben ist. Mit dieser Präsentation ist der Steuerungshinweis „Bitte anfassen!“ verbunden.

30 Diese Kürze ist auch den materialen Bedingungen der Lektüresituation geschuldet: die Objekttexte sind in ihrem Umfang beschränkt, da sie noch eindeutig als auf ein Objekt bezogen erkennbar sein müssen. 


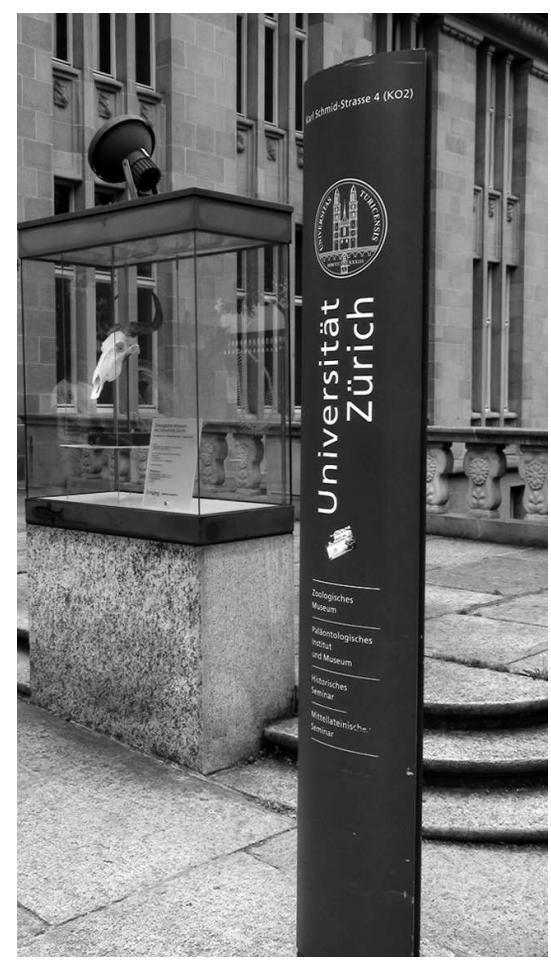

Abb. 9: Einzelne Stele vor dem Museumseingang

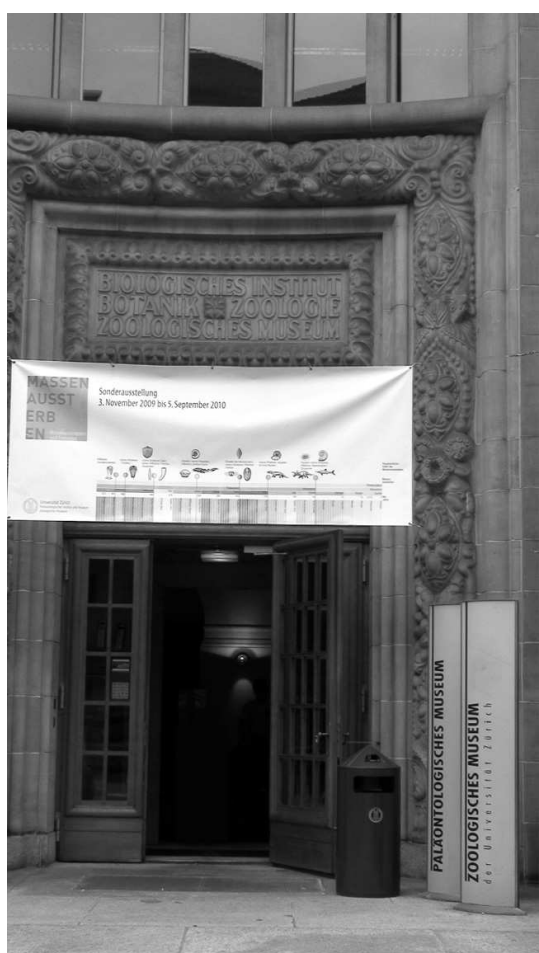

Abb. 10: Museumseingang

Wissensabhängig ist auch in diesem Vorkommen von Funktionshinweisen auf den Bereich der Wissenschaft ein Aspekt der Musterhaftigkeit von Objekttexten zu erkennen. Objekttexte sind nicht einfach Beschreibungstexte beliebiger Objekte. Es sind wissenschaftliche Beschreibungen für innerhalb des Wissenschaftssystems relevante Objekte. ${ }^{31}$

\subsection{Die Musterhaftigkeit der Intertextualitätshinweise}

Intertextualitätshinweise verweisen darauf, welche anderen Texte der Leser mit dem Gelesenen in Verbindung bringen soll, wobei es sich ebenso um einzelne, präzise identifizierte Texte handeln kann wie um ganze Textwelten: eine Vielzahl von durch ihr Thema, ihre Funktion oder ihren institutionellen Kontext miteinander verbundener Texte.

31 Diese Tatsache wird deutlich, wenn man Objekttexte beispielsweise mit Schildern mit Detailinformationen zu ausgestellten Möbelstücken vergleicht, wie man sie in Möbelhäusern findet. 
In den hier betrachteten Objekttexten findet man keine expliziten Hinweise auf andere (Einzel-)Texte. Auf diese Weise werden die in den Objekttexten vorgenommenen Informationen zu den Exponaten und ihrer Klassifizierung nicht als Ergebnisse wissenschaftlicher Entscheidungen oder als Positionen in einem wissenschaftlichen Diskurs präsentiert, sondern als einfache Tatsachen, die ihren Gültigkeitsanspruch nicht durch die Angabe von Entscheidungskriterien oder Quellen untermauern müssen. ${ }^{32}$ Einen Hinweis auf die Textwelt der Wissenschaft gibt jedoch implizit die notorische Verwendung griechisch-lateinischen Vokabulars, die vertrautheitsabhängig auf wissenschaftliche Disziplinen wie Paläontologie oder Geologie und deren Textwelten bezogen werden kann. Weitere vertrautheitsbasierte Intertextualitätshinweise lassen sich aus den Objekttexten gewinnen, wenn man in den zweigliedrigen Bezeichnungen von Zeile 1 einen Hinweis auf das auf Carl von Linné zurückgehende System der biologischen Nomenklatur entdeckt (z. B. „Acanthoceratites spinosus“) und so die Verbindung zwischen dem Gelesenen und der Textwelt (paläo-)biologischer Wissenschaft mit ihren zentralen Klassifikationsbemühungen herstellen kann; oder wenn man die offiziellen Schweizer Kantonskürzel wiedererkennt und von ihnen auf die Textwelten der staatlich normierten geographischen Referenzen (nicht die der alltäglichen Orientierung) als die relevanten Interpretationshintergründe schließt.

Schließlich lassen sich in unserem Korpus wahrnehmungsbasierte Hinweise entdecken, die sich erst durch unsere Vertrautheit mit der Institution Museum und ihren gestalterischen Traditionen erschließen lassen, und die es uns erlauben, in der typischen (d.h.: musterhaften) Platzierung von Schrift und Objekten in Glasvitrinen einen Hinweis auf museale Textwelten mit all den dort erwartbaren Texten und Textsorten $\mathrm{zu}$ lesen.

\subsection{Die Musterhaftigkeit der Objekttexte}

Die Analyse unseres kleinen Textkorpus hat exemplarisch gezeigt, wie man ausgehend von der Auswertung einzelner Textualitätshinweise, die dem Leser in der Lektüresituation (wahrnehmungs-, sprach- oder vertrautheitsbasiert) zur Verfügung stehen, Schritt für Schritt zu dem komplexen, ganzheitlichen Bild der Musterhaftigkeit einer Textsorte gelangen kann. Die Musterhaftigkeit der Textsorte als Ganze setzt sich

32 Dazu passt die Beobachtung, dass in den Objekttexten die Gewissheit des präsentierten Wissens nicht infrage gestellt wird (etwa durch Fragezeichen hinter einer Klassifikation, durch Modalwörter wie wahrscheinlich, vermutlich usw.). - Allerdings muss eingeräumt werden, dass das nur auf die Objekttexte zutrifft. In manch längeren Vitrinentexten werden vergangene wissenschaftliche Debatten durchaus thematisiert. 
zusammen aus der Musterhaftigkeit der einzelnen Textualitätsmerkmale und somit der auf sie gerichteten Hinweise. So gehört zur Musterhaftigkeit der Objekttexte

- die Musterhaftigkeit ihrer Abgrenzungshinweise: eine Abgrenzung durch das Mittel des ,leeren Raums', begrenzt durch ein Exponat einerseits und eine Vitrinenwand andererseits;

- die Musterhaftigkeit ihrer Gliederungshinweise: eine differenzierte, vor allem durch wahrnehmungsbasierte Hinweise angezeigte Binnenstruktur, sowohl, was den Objekttext als textuelle Ganzheit angeht, als auch, was seine Einordnung im Rahmen der Textsammlung Vitrine angeht, die untrennbar zur Musterhaftigkeit der Objekttexte dazugehört;

- die Musterhaftigkeit ihrer Verknüpfungshinweise mit der zentralen Rolle der Beziehung zwischen dem Objekttext und ,seinem' Objekt sowie zwischen dem Objekttext und allem, was sich um ihn herum in der Textsammlung Vitrine befindet;

- die Musterhaftigkeit des Textthemas, das durch Synonymie- und Teil-Ganzes-Relationen vorangebracht wird, worin sich zentrale wissenschaftliche Aktivitäten der Paläontologie spiegeln, sowie die herausragende Rolle der Ausstellungsstücke bei der Herstellung des Textthemas;

- die Musterhaftigkeit der Textfunktion, in der sich Darstellungs- und Reflexionsnützlichkeit auf spezifische Art und Weise mit dem Funktionsbereich Wissenschaft verbinden;

- die Musterhaftigkeit der intertextuellen Bezüge, der die Objekttexte auf die Welt der musealen Texte bezieht, allerdings ohne einen Dialog mit diesen Texten zu inszenieren (durch Verweise auf konkrete Einzeltexte, durch die Erörterung fremder Aussagen oder Klassifikationen usw.).

Aus der Musterhaftigkeit der einzelnen Textualitätshinweise und der durch sie hervorgebrachten Textualitätsmerkmale ergibt sich eine übergeordnete Musterhaftigkeit der Textsorte - hier: der Objekttexte im Paläontologischen Museum Zürich -, die vom Alltagsleser bei der Bestimmung der Textsortenzugehörigkeit einzelner Textexemplare ganzheitlich ausgewertet wird.

Die hier zusammenfassend dargestellte Musterhaftigkeit der Objekttexte ist ausgehend von einem begrenzten Korpus rekonstruiert worden. Allerdings lässt sich die Reichweite der einzelnen Aussagen problemlos erweitern, indem man das Korpus auf Texte aus anderen Museen (anderen naturhistorischen Museen, anderen Museumstypen) ausdehnt. Hierin liegt ein Vorteil der von dem Konzept der Textualitätshinweise ausgehenden Textsortenanalyse. Denn aus dieser Sichtweise sind nicht 
mehr Texte als Ganzes musterhaft, sondern die Hinweise, die die Texte konstituieren. Damit lassen sich Textsorten verschiedener Reichweite, ,gute' und ,schlechte' Fälle einer Textsorte, unterschiedliche Textsorten und ihre Mischungen etc. wesentlich detaillierter zueinander in Beziehung setzen, und es können Unterschiede und Gemeinsamkeiten zwischen Textsorten, Formen der strategischen Nutzung von Textsorten oder auch die Mischung mehrerer Textsortenkonventionen in einem Text präzise beschrieben werden. Kurz: Versteht man Musterhaftigkeit von Textsorten nicht als globales Phänomen, sondern als das Resultat der Musterhaftigkeit einzelner Textualitätshinweise, dann werden wesentlich anspruchsvollere Analysen von Musterhaftigkeit möglich.

Eine weitere allgemeine Konsequenz für die Analyse von Textsorten hat sich aus der exemplarischen Analyse der Objekttexte ergeben. Es zeigte sich nämlich, dass die Textsortenanalyse (wie die textlinguistische Analyse allgemein), notwendigerweise Hinweise aus drei „Quellen" berücksichtigen muss, um unseren Umgang mit Texten und Textsorten adäquat zu rekonstruieren: Wahrnehmung, Sprache und Vertrautheit. Gerade die oftmals vernachlässigte Textualitätsquelle der Wahrnehmung hat sich hier durchgehend als wichtiger Aspekt der Musterhaftigkeit der Objekttexte erwiesen. Das hat Folgen für die Daten, die für die Textsortenanalyse herangezogen werden müssen: Es reicht eben nicht aus, den Wortlaut von Texten zu analysieren. Vielmehr sollen die Daten möglichst viel von dem, was dem Leser während der Lektüresituation für seine alltagsweltliche Textanalyse zur Verfügung stand, der Analyse zugänglich machen. Damit rückt die Lektüresituation, nicht der Text in den Fokus der textlinguistischen Analyse. Dass diese Verschiebung von Nutzen sein kann, hat die Beispielanalyse des vorigen Abschnitts belegt: Geht man von vornherein vom Objekttext als der relevanten Analyseeinheit aus, dann kann man nicht mehr sehen, dass eine seiner Besonderheiten gerade darin liegt, keine völlig autarke textuelle Einheit zu sein. Das ist erst zu erkennen, wenn man bei der Analyse davon ausgeht, was sich aus den Signalisierungen in der Lektüresituation ergibt. Dann kann man - wie hier beispielhaft gezeigt wurde - erkennen, dass und wie Objekttexte musterhaft in ein komplexes Geflecht von Relationen innerhalb der Textsammlung Vitrine eingebettet sind.

\section{Ergebnisse der exemplarischen Textsortenanalyse}

Welche weiter reichenden Erkenntnisse über Textsorten lassen sich aus der Beispielanalyse gewinnen?

Zunächst einmal ist Textsortenzugehörigkeit nicht einfach gegeben. Sie wird beim Lesen konstruiert, und zwar dadurch, dass wir bei der 
Lektüre eine große Bandbreite von Hinweisen auf Textsortenzugehörigkeit auswerten. Es ist die Leistung dieser Hinweise, dass uns in unserem Lektürealltag die Zuordnung von einzelnen Texten zu Textsorten im Allgemeinen als unproblematisch erscheint. Die Aufgabe der Textsortenlinguistik ist es, auf den routinehaften, meist unter unserer Bewusstseinsschwelle ablaufenden Prozess der Auswertung dieser Hinweise zu fokussieren und so unseren alltäglichen Umgang mit Textsorten einer eingehenden, systematischen Analyse zugänglich zu machen. Mit anderen Worten: Es gilt, mit Hilfe detaillierter Analysen die Hinweise auf Textualität zu rekonstruieren, die die Leser aus der Lektüresituation heraus nutzen können, um etwas als Exemplar einer bestimmten Textsorte zu identifizieren und seinen konkreten kommunikativen Sinn zu bestimmen.

Trotz ihrer ähnlichen Bezeichnung sind Textualitätshinweise nicht mit den Textualitätskriterien von de Beaugrande und Dressler (1981) gleichzusetzen. Einerseits, weil die Textualitätshinweise konsequent vom Text und seinen kommunikativen Erscheinungsformen her gedacht sind - ihr Vorliegen muss immer ausgehend von dem in der Lektüresituation Wahrnehm- und Aktivierbaren beurteilt werden, und nicht einmal mit Bezug auf ein Urteil des Rezipienten (,Akzeptabilität"), ein anderes Mal mit Bezug auf mentale Zustände des Produzenten (,Intentionalität") oder die Situation (,Situationalität"). Zum anderen, weil es bei den Textualitätshinweisen nicht darauf ankommt, Texte von NichtTexten abgrenzen zu können (vgl. dazu Vater 2001). Tatsächlich hat die Analyse der Objekttexte gezeigt, dass es durchaus zur Textsortenspezifik dazugehören kann, dass das eine oder andere Textualitätsmerkmal nur schwach realisiert ist (etwa das Merkmal der Verknüpfung, v.a. wenn man nur die Textualitätsquelle Sprachlichkeit heranzieht), und dass diese Tatsache weder für das Verständnis der Texte noch für die Frage „Sind das überhaupt Texte?“ von Bedeutung zu sein scheint. Ihre Relevanz für die Analyse gewinnen die Textualitätshinweise also nicht daraus, dass sie die Identifikation von Nicht-Texten erlaubten. Vielmehr geben sie uns ein Instrumentarium in die Hand, das empirisch vorkommende Texte in ihrem unproblematischen wie problematischen Funktionieren beschreiben kann. Textualitätshinweise erlauben es zu verstehen, was die ganz spezifische Kommunikationsleistung eines Texts ist und wie er seinen Lesern seine Leistung signalisiert.

Textsortenzugehörigkeit kann durch unterschiedliche Typen von Musterhinweisen angezeigt werden: Zum einen können Musterhinweise metakommunikativer Natur sein (ich habe weiter oben, ohne Anspruch auf Terminologisierung, von „dezidierten Musterhinweisen“ gesprochen). Zum anderen kann es sich um Hinweise auf Textualität handeln, die erst dann zu Musterhinweisen werden, wenn sie vertrautheitsabhän- 
gig als musterhaft ausgeprägt wahrgenommen werden. Textsorten ergeben sich also aus expliziten Textsortenhinweisen und der musterhaften Ausprägung anderer Textualitätshinweise.

Dabei kann jedes einzelne Textualitätsmerkmal musterhaft sein. Das lässt sich schon an den alltagssprachlichen Bezeichnungen für Textsorten ablesen, die oftmals die Musterhaftigkeit eines spezifischen Merkmals in den Vordergrund rücken, etwa die spezifische Form der Abgrenzung (Verkehrsschild, Einkaufszettel), Formen der Verknüpfung (Erzählung, Wegbeschreibung), des Themas (Wegbeschreibung), der Funktion (Bewerbungsschreiben, Glückwunschkarte) oder der Intertextualität (Replik). Wie an der exemplarischen Analyse der Objekttexte zu sehen war, ist es aber häufiger die spezielle Kombination der Musterhaftigkeit mehrerer, eventuell aller Textualitätsmerkmale, die für die Wiedererkennbarkeit einer Textsorte Ausschlag gebend sein dürfte.

Wenn es aber stimmt, dass Textsorten als Ergebnis der Musterhaftigkeit allgemeiner Textualitätshinweise zu verstehen sind, bedeutet das, dass Leser die Frage, ob eine Anzahl sinnlich wahrnehmbarer Erscheinungsformen als Text zu verstehen ist (und nicht als bloße Ansammlung von zufälligen Strichen oder Wörtern $)^{33}$ und die Frage, welcher Textsorte ein Text zuzurechnen ist, ausgehend von den gleichen Textualitätshinweisen, beantworten. Für die Textlinguistik heißt das, zumindest wenn sie sich zum Ziel setzt, unseren alltäglichen Umgang mit Texten systematisch zu rekonstruieren: die Begriffe Text und Textsorte sind nicht unabhängig von einander zu bestimmen. Die Frage nach dem ,Wesen' des Texts und die Frage nach den Kriterien der Textsortenbestimmung, die in der Textlinguistik bisher getrennt voneinander behandelt worden sind, werden aus der Perspektive der Textualitätshinweise zu einer einzigen Frage: der Frage nach den Textualitätshinweisen und ihrer Musterhaftigkeit.

\section{Literaturverzeichnis}

de Beaugrande, Robert Alain u. Wolfgang U. Dressler: Einführung in die Textlinguistik. Tübingen 1981.

Bergmann, Jörg: Ethnomethodologie und Konversationsanalyse. Kurseinheit 1. Hagen 1988.

33 Dass diese Problematik auch abseits textlinguistischer Fragestellungen relevant sein kann, zeigt beispielhaft der immer wieder diskutierte Fall von Auberginen, in denen das Wort „Allah" in arabischer Schrift zu sehen sein soll (<http://theworldof-islam.blogspot.com/ 2009/01/eggplant-with-name-of-allah html $>$ (Stand: 01.12.2010)). - Diesen Hinweis verdanke ich Hiloko Kato. 
Borun, Minda u. Maryanne Miller: „To label or not to label.” In: Museum News 2/59 (1980), 64-67.

Bühler, Karl: Sprachtheorie. Die Darstellungsfunktion der Sprache. Jena 1934.

Cicourel, Aaron V.: „Discourse and Text: Cognitive and Linguistic Processes in Studies of Social Structure.“ In: Versus: Quaderni di Studi Semiotici 2/12 (1975), 33-84.

Daneš, František: „Zur linguistischen Analyse der Textstruktur.“ In: Folia Linguistica (1970), 72-78.

Dawid, Evelyn u. Robert Schlesinger: Texte in Museen und Ausstellungen: Ein Praxisleitfaden. Bielefeld 2002.

Garfinkel, Harold: „,Good“ organizational reasons for ,bad' clinical records.“ In: Studies in Ethnomethodology. Englewood Cliffs/New Jersey 1967, 186-207.

Graf, Bernhard: „Texte und Beschriftungen in Museumsausstellungen - zur Information des Besuchers oder zum Ruhm des Curators?" In: Museumsausstellungen. Planung - Design - Evaluation. Hg. v. Bernhard Graf u. Günther Knerr. München 1985, 66-73.

Gülich, Elisabeth: „Zum Zusammenhang von alltagsweltlichen und wissenschaftlichen ,Methoden“." In: Text- und Gesprächslinguistik. Ein internationales Handbuch zeitgenössischer Forschung. Gesprächslinguistik Halbbd. 2. Hg. v. Klaus Brinker, Gerd Antos, Wolfgang Heinemann u. Sven Frederik Sager. Berlin, New York 2001, 1086-1093.

Gumperz, John J.: Discourse strategies. Cambridge 1982.

Hausendorf, Heiko u. Wolfgang Kesselheim: Textlinguistik fürs Examen. Göttingen 2008.

Hutchby, Ian u. Robin Wooffitt: Conversation analysis. Principles, practices and applications. Cambridge 2008.

Jakobson, Roman: „Linguistik und Poetik.“ In: Strukturalismus in der Literaturwissenschaft. Hg. v. Heinz Blumensath. Köln 1972, 118-147.

Kesselheim, Wolfgang: „Wissensvermittlung in der Museumsausstellung als Herausforderung für die Linguistik." In: Wissenstransfer und Diskurs. Hg. v. Oliver Stenschke. Frankfurt/Main 2009, 245-266.

Kesselheim, Wolfgang: „Wechselspiele von ,Text ${ }^{\star}$ und ,Kontext ${ }^{\star}$ in multimodaler Kommunikation." In: Kontext und Texte. Soziokulturelle Konstellationen literalen Handelns. Hg. v. Peter Klotz, Paul R. Portmann-Tselikasu u. Georg Weidacher. Tübingen 2010, 327-343.

Kesselheim, Wolfgang: „Wissensvermittlung multimodal: Wie Museumsbesucher sich über den Inhalt einer Museumsvitrine verständigen." In: Fachsprache: Internationale Zeitschrift für Fachsprachenforschung, -didaktik und Terminologie. (demnächst).

Klein, Wolfgang u. Christiane von Stutterheim: „Quaestio und referentielle Bewegung in Erzählungen.“ In: Linguistische Berichte 109 (1981), 163-183.

Luckmann, Thomas: „Grundformen der gesellschaftlichen Vermittlung des Wissens: Kommunikative Gattungen." In: Sonderheft 27 der Kölner Zeitschrift für Soziologie und Sozialpsychologie (1986). Hg. v. M. Rainer Lepsius, Friedhelm Neidhardt u. Johannes Weiß, 191-211.

McManus, Paulette M.: „Oh, yes, they do: How museum visitors read labels and interact with exhibit texts.“ In: Curator 3/32 (1989), 174-189. 
Sacks, Harvey, Emanuel A. Schegloff u. Gail Jefferson: „A simplest systematics for the organization of turn-taking for conversation." In: Studies in the Organization of Conversational Interaction. Hg. v. Jim Schenkein. New York 1978, $7-55$.

Schenkein, Jim: Studies in the Organization of Conversational Interaction. New York 1978.

Schneemann, Peter: „Das ausgestellte Wort. Zum Umgang mit Texten im Museum." In: Museum als Medium - Medien im Museum: Perspektiven der Museologie. Hg. v. Hubert Locher, Bärbel Küster, Beat Wyss u. Angela Ziegler. München 2004, 28-43.

Smith, Dorothy E.: „The active text. Texts as constituents of social relations.“ In: Texts, facts, and feminity. Exploring the relations of ruling. Hg. v. Dorothy E. Smith. London 1990, 120-158.

Techtmeier, Bärbel: „Bezeichnungen von Textsorten im GWDS.“ In: Untersuchungen zur kommerziellen Lexikographie der deutschen Gegenwartssprache II. Hg. v. Herbert Ernst Wiegand. Tübingen 2005, 219-229.

Tripps, Manfred: „Too much to read - to little to see: Exhibitions techniques and the 2-D syndrome." In: Museological News 10 (1987), 203-210.

Vater, Heinz: Einführung in die Textlinguistik. Struktur und Verstehen von Texten. München 2001.

Weber, Traudel: „Warum darf das Tier mit unguligrader Lokomotion nicht einfach auf vier Hufen gehen? oder: Besucherfreundliche Texte in Museen und Ausstellungen." In: Texte in Ausstellungen. Hinweise und Anregungen für verständliche Formulierung und besucherfreundliche Gestaltung. Hg. v. Alfons W. Biermann. Köln 1995, 63-111.

Wolff, Stephan: „Textanalyse.“ In: Qualitative Methoden der Medienforschung. Hg. v. Ruth Ayaß u. Jörg Bergmann. Reinbek 2006, 245-273. 